\title{
Identifying potential heterosis in perennial ryegrass
}

\author{
J.R. O'CONNOR, T.B. LYONS, M.Z.Z. JAHUFER, M. FAVILLE and B.A. BARRETT \\ AgResearch Ltd, Grasslands Research Centre, Private Bag 11008, Palmerston North
}

Jessica.OConnor@agresearch.co.nz

\begin{abstract}
Plant breeders aim to develop cultivars of perennial ryegrass Lolium perenne L., (PRG) that better support New Zealand farmers through improved genetic potential for key traits such as seasonal dry matter yield. Hybrid vigour (heterosis) may be one way plant breeders can increase dry matter yields, and potentially lift the long term rate of genetic gain. We report evaluation of PRG full-sibling progeny for heterosis expressed under grazing. Parents were randomly sampled from sixteen cultivars, and for each cultivar combination within mid-season and late season classes, six pair crosses were made. In 2013, replicated single row trials of parent cultivar samples and progeny were sown at two sites near Palmerston North. For 2 years, prior to each grazing, growth score (GS) data were recorded to estimate dry matter yield of each plot. Progeny GS relative to parent average GS (mid-parent heterosis, MPH), and better parent GS (high-parent heterosis, HPH) were calculated on the basis of the mean GS over years and sites. Significant $(P<0.05)$ line effects were detected, as were site, season, and year interactions. For the medium flowering date progeny, MPH and HPH GS ranged from -19 to $23 \%$ and -22 to $18 \%$, respectively. For the late flowering date progeny MPH and HPH for GS ranged from -9 to $25 \%$ and -15 to $21 \%$, respectively. Some progeny exhibited significant $(\mathrm{P}<0.05) \mathrm{MPH}$ and $\mathrm{HPH}$. The five progeny with highest MPH values ranged from 14 to $23 \%$ and 10 to $25 \%$ for crosses within medium and late flowering classes, respectively. The same top progeny exhibited $\mathrm{HPH}$ ranging from 12 to $18 \%$ and 7 to $21 \%$ among medium and late flowering classes, respectively. A moderate, significant $(\mathrm{P}<0.05)$ negative correlation was observed between high GS of the better parent and the progeny's level of HPH. However there were notable exceptions to the trend. The GS of the best progeny entry was significantly $(\mathrm{P}<0.05)$ higher than the GS of the best parent entry, indicating the potential of hybrid vigour to lift the yield barrier in PRG. Investigation is needed of the genetic, wider biological, and economic context under which a hybrid breeding system may be justified.
\end{abstract}

\section{Introduction}

Cool season forage species are the basis of New Zealand's meat, wool, and dairy industries. Together with white clover (Trifolium repens L.), perennial ryegrass, (Lolium perenne L.; PRG) is a critical component of cost-efficient pastoral-based agriculture. Over time, plant breeders have developed PRG cultivars that add value to New Zealand farm businesses through improving herbage dry matter yields (Easton et al. 2001; Kerr 1987; Lee et al. 2012; Woodfield 1999). More recently, combining elite cultivars with novel, less animal toxic strains of fungal endophyte has extended the value of modern ryegrass cultivars (Johnson et al. 2013). However, compared with other species such as cereals, PRG has a relatively low rate of genetic gain for key traits (Brummer \& Casler 2014). This can be attributed to limitations imposed by genetic and physiological parameters (such as obligate outcrossing), and the constraints of minor species breeding programmes (Barrett et al. 2010; Brummer 1999; Easton et al. 2002; Woodfield 1999).

An option for plant breeders to increase dry matter yield potential and genetic gain may be to harness heterosis, also known as hybrid vigour. Heterosis is the genetic phenomenon whereby progeny deviate from their expected performance calculated from parent values for a trait, and is often focused on harvestable yield. Mid-parent heterosis (MPH) is the extent that progeny deviate from the average performance of both parents. High-parent heterosis (HPH) is the extent that progeny deviates from its better performing parent. High-parent heterosis is often used to assess the potential value of hybrid progeny for use in agriculture (Brummer 1999; Springer \& Stupar 2007).

Some self-compatible plant species have successfully exploited heterosis for hybrid cultivar development. Most notably, the reproductive structures in maize (Zea mays) enable convenient self and cross pollination. This has enabled breeders to generate inbred lines and carry out crosses to evaluate their specific combining ability associated with heterosis effects, resulting in the development of successively improved hybrid cultivars over a number of decades (Betrán 2007; Springer \& Stupar 2007).

Perennial ryegrass however is a highly selfincompatible species, making the development of completely hybrid populations challenging. Brummer (1999) and Foster (1973) suggest a "semi-hybrid" concept for outcrossing species. Here, parents are identified that exhibit high levels of heterosis, and the population sources of the parents are polycrossed. The resulting population progeny are expected to be half 
inter-pollination crosses that express heterosis, and half derived from intra-pollination crosses.

Barrett et al. (2010) previously explored the potential for heterosis in PRG in New Zealand. The single site study showed cultivar-by-cultivar pair cross progeny performed better than progeny of cultivar crosses with unimproved or non-adapted parents. On average, low levels of HPH (mean of 1.6\%) were observed, with only a few semi-hybrids exhibiting significant hybrid vigour. However, heterosis levels were highly influenced by season with the estimated yield of the best semi-hybrid progeny between 7\% (autumn) to 19\% (spring) better than its better parent.

The aim of our research was to further investigate the potential to identify patterns of heterosis among PRG cultivars adapted to New Zealand. This was achieved by evaluation of seasonal growth of full-sib families and their parents in multi-site experiments under grazing management for 2 years in the Manawatu region.

\section{Materials and Methods \\ Trial sites}

The two field sites used for this experiment were AgResearch Aorangi farm, Rongotea $\left(40^{\circ} 34^{\prime} \mathrm{S}\right.$, $175^{\circ} 47^{\prime} \mathrm{E}$ ); and AgResearch Grasslands Research Centre, Palmerston North $\left(40^{\circ} 21^{\prime} \mathrm{S}, 175^{\circ} 37^{\prime} \mathrm{E}\right)$. The soil type for both sites is Kairanga silt loam (Cowie 1978). Soil tests to $7.5 \mathrm{~cm}$ indicated $\mathrm{pH}$ of 5.0 and 5.5, Olsen P of 36 and $51 \mu \mathrm{g} / \mathrm{ml}$, and sulphur levels of 7 and $56 \mathrm{ppm}$ for Aorangi and Grasslands, respectively. Both sites were prepared for sowing by ploughing, harrowing and rolling the soil before sowing with a mechanical drill.

The Aorangi site had total annual rainfall of $891 \mathrm{~mm}$ and $808 \mathrm{~mm}$ for 2013 and 2014, respectively. The Grasslands site had total annual rainfall of $847 \mathrm{~mm}$ and $763 \mathrm{~mm}$ for 2013 and 2014, respectively.

\section{Generation of full sib families}

Ten medium heading date and six late heading date perennial ryegrass cultivars of known endophyte status (Table 1) were used in this study to make pair crosses within each heading date class. For each within-class cultivar combination, six sets of pair crosses were made. All pair crosses were conducted in a glasshouse during summer 2012/13, using potted plants. Each cross was isolated by placing an isolation vyleen bag over all spikes from the two parental plants growing in adjacent pots, before flowering, with mechanical stimulation to achieve pollen transfer. To account for endophyte differences between cultivars, seed from each parent within each pair cross was harvested separately, giving rise to twelve progeny samples per cultivar combination. These samples were bulked by maternal group.

\section{Field trials and measurements}

Maternal progeny (120 in total), and seed from each of the 16 parent cultivars were sown in late autumn 2013 as $1 \mathrm{~m}$ long single row plots. Plots were sown at a rate of $13 \mathrm{~kg} / \mathrm{ha}$ using a mechanical planter. At each site, a row-column experimental design with three replicates was used. All trial maintenance protocols were conducted according to standard pasture management on each research farm.

As an estimate of dry matter yield, seasonal growth score (GS) assessments began in mid-August 2013. Prior to grazing, GS data were collected using a visually assessed scale of 1 (poorest in trial) to 9 (best in trial).

The trials were grazed by sheep at both sites. Following each GS assessment, plots were grazed for approximately 1 day then mechanically topped to a uniform height of 4 $\mathrm{cm}$. When soil moisture was adequate, nitrogen fertiliser was applied shortly after each grazing at a rate of 25-30 $\mathrm{kg} \mathrm{N} / \mathrm{ha}$ in autumn and spring.

Heterosis levels were calculated as the percent change in progeny GS relative to the average of the two parents (mid-parent heterosis, MPH), or relative to the better parent (high-parent heterosis, HPH). Analysis of the data was conducted using the Residual Maximum Likelihood (REML) and correlation functions in GenStat. For REML, a mixed linear model was used with full sib family/cultivar entries and seasons as random effects and sites and years as fixed effects.

\section{Results}

The analysis of growth across the Aorangi and Grasslands sites indicated significant $(\mathrm{P}<0.05)$ genotypic variation for GS among the parent and progeny lines (Table 2).

Table 1 The ten medium and six late flowering perennial ryegrass cultivars and their fungal endophyte strain used in this study to assess heterosis potential using single row plots of source cultivars and paircross progeny under grazed field conditions in a multi-site, multi-year trial in the Manawatu.

\begin{tabular}{cccc}
\hline \multicolumn{2}{c}{ Medium } & \multicolumn{2}{c}{ Late } \\
\hline Cultivar & Endophyte & Cultivar & Endophyte \\
\hline Arrow & AR1 & Abermagic & AR1 \\
Commando & nil & Expo & AR1 \\
Extreme & nil & Matrix & WT \\
Hillary & AR1 & One50 & WT \\
Kamo & AR37 & Revolution & AR1 \\
Kingston & WT & Ultra & AR1 \\
Pacific & WT & & \\
Request & AR37 & & \\
Samson & WT & & \\
Stella & AR1 & & \\
\hline
\end{tabular}


Site and year effects were not significant, however there were significant $(\mathrm{P}<0.05)$ line-by-year, line-by-site and line-by-season interactions. The genotypic component of variation among the lines was much higher than the interaction components.

Both medium-flowering date and late-flowering date progeny had greater overall mean growth score. Midflowering and late flowering progeny had greater means than their parents by $2.4 \%$ and $3.2 \%$ respectively (data not shown). Both medium and late flowering date progeny also showed significant $(\mathrm{P}<0.05)$ genotypic variation for MPH and HPH (Table 3). For the medium-flowering date

Table 2 Sources of variation with significant $(\mathrm{P}<0.05)$ variance, among the medium and late flowering perennial ryegrass cultivar parents and their full sib progeny estimated from REML analysis of seasonal growth scores across 2 years, seasons and two sites.

\begin{tabular}{lc}
\hline Source of variation & Variance component \\
\hline Line & $0.16^{*}$ \\
Line-by-Year interaction & $0.02^{*}$ \\
Line-by-Site interaction & $0.05^{\star}$ \\
Line-by-Season interaction & $0.07^{*}$ \\
Within Season sampling error & $0.15^{*}$ \\
Pooled error & $0.80^{*}$ \\
\hline
\end{tabular}

${ }^{*} \mathrm{P}<0.05$ progeny, MPH and HPH GS ranged from -19 to $23 \%$ and -22 to $18 \%$, respectively, and for the late flowering date progeny MPH and HPH ranged from -9 to $25 \%$ and -15 to $21 \%$, respectively (data not shown). Ranked on $\mathrm{HPH}$, the top five progeny expressing heterosis for the midflowering group exhibited MPH 14 to $23 \%$ greater than the average GS of their parents. For the same progeny, HPH ranged from $12 \%$ to $18 \%$ above their better parent (Table 3). The top five progeny expressing heterosis for late-flowering group exhibited MPH 10 to $25 \%$ greater than the average GS of their parents (Table 3). For the same progeny HPH ranged from 7 to $21 \%$ above their better parent.

Increasing heterosis levels in the progeny did not necessarily equate to better ranking progeny in the experiment. Of the 89 medium flowering date full sib progeny, the progeny with the highest HPH ranked 28 for mean GS across sites and years. However, in the late flowering date group, the highest ranking full sib progeny was also the top performer for GS with a mean of 7.25 , compared to the best non-hybrid entry with a mean of 6.84 . This progeny was significantly $(\mathrm{P}<0.05)$ higher by $6 \%$ in mean GS than the best performing cultivar in this trial.

The data indicated a trend whereby increasing high parent GS is negatively correlated with HPH (Figure 1). The correlations were significant $(\mathrm{P}<0.05)$ within medium flowering $\left(\mathrm{R}^{2}=0.09\right)$ and late flowering $\left(\mathrm{R}^{2}=\right.$ $0.44)$ classes. However, there are exceptions to that trend, with the best entries in the experiment being progeny rather than parents.

Table 3 The five greatest HPH values for each flowering class (medium and late), with MPH, Growth Score (GS), and ranking observed amongst full sib progeny within medium and late flowering perennial ryegrass across 2 years and two locations in the Manawatu region, relative to their paternal and maternal parent GS means. GS was scored on a 1: poor to 9: excellent scale. Growth Score LSD $(\mathrm{P}<0.05)=0.4$.

\begin{tabular}{cccccc}
\hline \multicolumn{5}{c}{ Medium Flowering Class } \\
\hline HPH (\%) & MPH (\%) & Paternal Parent GS & Maternal Parent GS & Progeny GS & Progeny GS Rank \\
\hline 17.8 & 22.6 & 5.14 & 4.73 & 6.05 & 28 \\
14.1 & 14.7 & 5.54 & 5.60 & 6.39 & 4 \\
13.3 & 14.1 & 4.80 & 4.73 & 5.44 & 62 \\
13.2 & 13.8 & 5.60 & 5.54 & 6.34 & 5 \\
12.1 & 16.3 & 5.14 & 5.54 & 6.21 & 12 \\
\hline
\end{tabular}

Late Flowering Class

\begin{tabular}{cccccc}
\hline HPH (\%) & MPH (\%) & Paternal Parent GS & Maternal Parent GS & Progeny GS & Progeny GS Rank \\
\hline 20.7 & 24.9 & 6.00 & 5.60 & 7.25 & 1 \\
13.4 & 17.4 & 5.60 & 6.00 & 6.81 & 3 \\
9.6 & 12.3 & 6.20 & 5.90 & 6.79 & 4 \\
8.7 & 11.4 & 6.00 & 6.30 & 6.85 & 2 \\
7.1 & 9.9 & 5.90 & 5.60 & 6.32 & 16 \\
\hline
\end{tabular}




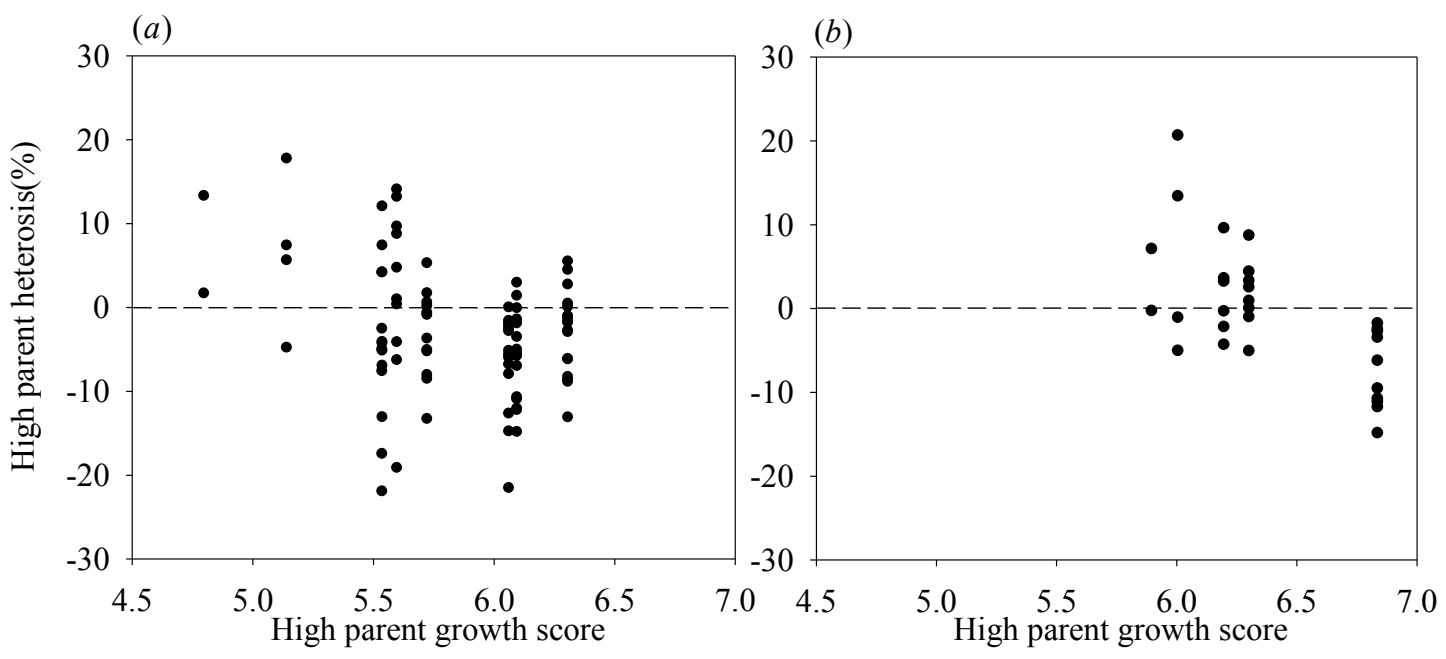

Figure 1 High-Parent Heterosis versus better parent growth score for (a) medium flowering and (b) late flowering full-sib progeny within each pair cross in a 2 year study of heterosis in perennial ryegrass at two field sites in the Manawatu region. In both cases there is a significant negative correlation between HPH and GS of the better parent, however there are individual progeny that are exceptions to that trend in each class.

\section{Discussion}

Growth scores were used to assess the rows in this preliminary trial to get an indication of heterotic effects occurring. Smith et al. (2001) confirmed that growth score data to assess perennial ryegrass rows was significantly correlated with dry matter cuts, and visual scoring is a common and successful practice in plant breeding protocols. In future trials, it is planned to use cutting to identify dry matter yield differences in heterotic progeny.

The significant $(\mathrm{P}<0.05)$ genotypic variation estimated indicates the potential additive and non-additive genetic variation available. Comparison of full sib family and parental mean seasonal growth indicated the presence of full sib families with GS higher than their pair cross parent cultivars. In the medium and late flowering groups the top five full sib progeny had high parent heterosis ranging from $12.1 \%-17.8 \%$ and $7.1 \%-20.7 \%$, respectively. The expression of high parent heterosis detected in this study is consistent with results reported by Barrett et al. (2010), and Foster $(1971,1973)$. The levels of high parent heterosis measured in this study were higher than those reported by Barrett et al. (2010), but lower than those found by Foster (1971) under spaced plant conditions (38\% heterosis). When identifying heterosis that may be useful, it is important to evaluate full sib families and their parents in environments similar to those on farm, such as sward plots as used by Barrett et al. (2010). However there are limitations with swards for breeders, such as the small amounts of seed available to conduct multi-site evaluation among large numbers of parent combinations. Using rows is the next best option, and there is evidence that row yields correlate well with yields in swards for perennial ryegrass (Smith et al. 2001).
Comparison of parent and full sib progeny GS indicated a moderate negative association between parent GS and HPH in full sib progeny. This trend is more obvious in late flowering types, which also had the higher performing parents. However, there were within any parent GS category a range of HPH levels, and the best line in the trial was a hybrid, indicating scope to identify combinations expressing heterosis that can outperform parents. In our study the late flowering full sib progeny with the highest GS had a mean score of 7.25. This was significantly $(\mathrm{P}<0.05)$ greater than the highest performing cultivar which had a mean seasonal growth score of 6.84 (LSD=0.4). It was also observed that the highest performing cultivar was not a parent of the highest performing progeny, a further indication of heterosis in some combinations.

The five progeny with highest HPH in the medium flowering group had relatively low overall mean GS ranking compared to the other entries. However, in the late flowering group, the progeny with the highest HPH was also the best performing entry.

The limited sample of six parents per cultivar per cross, and use of a random sample of the cultivar rather than parent clones are limitations of the study. However, the variation in HPH and trends identified of increasing parent GS relating to decreasing HPH, with exceptions, is consistent with theoretical and empirical evidence (Barrett et al. 2010; Brummer 1999).

In this study the line effects were stronger than the interactions for line $\times$ site and line $\times$ year, and there were also genotype-by-environment $(\mathrm{G} \times \mathrm{E})$ interactions. The importance of $\mathrm{G} \times \mathrm{E}$ interaction in perennial ryegrass breeding in New Zealand was discussed by Lee et 
al. (2012). In our study, results from analysis of the performance of the medium and late flowering perennial ryegrass full sib progeny and their parents, based on seasonal growth across two sites, showed significant $(\mathrm{P}<0.05)$ line-by-site interaction. This indicated a change in relative performance of the full sib progeny and parents across the two evaluation sites Aorangi and Grasslands. Also, the significant $(\mathrm{P}<0.05)$ line-by-season interaction effects, indicated a re-ranking of lines due to seasonal effects. These results are similar to those of Barrett et al. (2010) who found seasonality affected HPH expression in assessments of semi-hybrid perennial ryegrass sward plots in New Zealand.

Further work is needed to explore the differences in seasonal responses of semi-hybrids, assess endophyte effects, better characterise the patterns and effects of relatedness among parents, conduct an economic analysis of the FVI framework, and understand the possible implications of seed production of an F1 hybrid or semihybrid cultivar of perennial ryegrass in order to justify a transition to hybrid or semi-hybrid approaches to breeding.

\section{ACKNOWLEDGEMENTS}

The research was funded by Pastoral Genomics, a joint venture co-funded by DairyNZ, Beef + Lamb New Zealand, Dairy Australia, AgResearch Ltd, New Zealand Agriseeds Ltd, Grasslands Innovation Ltd, DEEResearch, and the Ministry of Business, Innovation and Employment (New Zealand). Special thanks to research farm managers Steve Lees and Trevor Holloway, and Bryce Clark for technical assistance.

\section{REFERENCES}

Barrett, B.A.; Turner, M.A.; Lyons, T.B.; Rolston, M.P.; Easton, H.S. 2010. Evaluation of semi-hybrid perennial ryegrass populations. Proceedings of the New Zealand Grasslands Association 72: 11-16.

Betrán, F.J. 2007. Industry highlights: Hybrid breeding in maize. pp. 491-497. In: Principles of plant genetics and breeding. Ed. Acquaah, G. Blackwell, United Kingdom.

Brummer, E.C. 1999. Capturing heterosis in forage crop cultivar development. Crop Science 39: 943-954.

Brummer, E.C.; Casler, M.D. 2014. Cool-season forages. pp. 33-52. In: Yield gains in major U.S. field crops. Eds. Smith, S.; Diers, B.; Specht, J.; Carver, B. American Society of Agronomy, Inc., Crop Science Society of America, Inc., and Soil Science Society of America, Inc.,
Cowie, J.D. 1978. Soil and agriculture of Kairanga County, North Island, New Zealand. Soil Bureau, N. Z. S. 33:

Easton, H.S.; Aymes, J.M.; Cameron, N.E.; Green, R.B.; Kerr, G.A.; Norriss, M.; Stewart, A.V. 2002. Pasture plant breeding in New Zealand: where to from here? Proceedings of the New Zealand Grasslands Association 64: 173-179.

Easton, H.S.; Baird, D.B.; Cameron, N.E.; Kerr, G.A.; Norriss, M.; Stewart, A.V. 2001. Perennial ryegrass cultivars: herbage yield in multi-site plot trials. Proceedings of the New Zealand Grasslands Association 63: 183-188.

Foster, C.A. 1971. Interpopulation and intervarietal hybridization in Lolium perenne breeding: heterosis under non-competitive conditions. Journal of Agricultural Science 76: 107-130.

Foster, C.A. 1973. Prospects for the development of F1 hybrid varieties of perennial ryegrass (Lolium perenne) and other herbage grasses. Journal of Agricultural Science 81: 33-37.

Johnson, L.J.; De Bonth, A.C.M.; Briggs, L.R.; Caradus, J.R.; Finch, S.C.; Fleetwood, D.J.; Fletcher, L.R.; Hume, D.E.; Johnson, R.D.; Popay, A.J.; Tapper, B.A.; Simpson, W.R.; Voisey, C.R.; Card, S.D. 2013. The exploitation of Epichloë endophytes for agricultural benefit. Fungal diversity 60: 171-188.

Kerr, G.A. 1987. Evaluation of four perennial ryegrass cultivars in New Zealand. Proceedings of the New Zealand Grasslands Association 48: 137-141.

Lee, J.M.; Matthew, C.; Chapman, D.F. 2012. Perennial ryegrass breeding in New Zealand: a dairy industry perspective. Crop and Pasture Science 63: 107-127.

Smith, K.F.; Tasneem, M.; Kearney, G.A.; Reed, K.F.M.; Leonforte, A. 2001. Evaluation of herbage yield in a forage grass breeding program: comparison of visual rating versus measurement in single-row plots or swards. Australian Journal of Experimental Agriculture 41: 1161-1166.

Springer, N.M.; Stupar, R.M. 2007. Allelic variation and heterosis in maize: how do two halves make a whole? Genome Research 17: 264-275.

Woodfield, D.R. 1999. Genetic improvements in New Zealand forage cultivars. Proceedings of the New Zealand Grasslands Association 61: 3-7. 
